\title{
Pharmacoinformatic Studies on 4-Thiazolyl-phenoxy Tail Containing Indanyl Acetic Acid Derivatives as PPAR-Pan Agonists as Potent Anti-Diabetic Agent
}

\author{
Neha Verma*, Usha Chouhan \\ Department of Mathematics, Bioinformatics and Computer Applications, MANIT, Bhopal, Madhya Pradesh, INDIA.
}

\begin{abstract}
Aim: The increasing incidences of type 2 diabetes mellitus, represents a considerable public health problem and characterized by loss in sensitivity of tissues to insulin which can be restored by activation of Peroxisome Proliferator-Activated Receptors (PPARs). The present work takes in consideration for the development of PPAR agonists, which can activate PPARs and is expected to lower LDL cholesterol and triglycerides, raise HDL cholesterol and normalize hyperglycaemia. Materials and Methods: Quantitative Structure-Activity Relationship (QSAR) study is performed by means of Multiple Linear Regression (MLR) analysis on a set of indanyl acetic acid derivatives followed by ADMET prediction and Docking Studies. Results: A good correlation is found by regression analysis between the observed and predicted activities as evident by their $R^{2}(0.81)$, $\mathrm{Q}^{2}(0.81)$ and $R^{2}$ pred (0.86) for PPAR $\alpha$ and $R^{2}(0.66), \mathrm{Q}^{2}(0.66)$ and $R^{2}$ pred (0.90) for PPAR $\delta$ and $R^{2}(0.82), \mathrm{Q}^{2}(0.77)$ and $R^{2}$ pred (0.58) for PPAR $\gamma$ respectively. Molecular docking of the ligands qualifying all the Drug Likeness properties to the proteins PPAR $\alpha$ (PDB ID: 3ET1), PPAR (PDB ID: 3ET2) and PPAR $\gamma$ (PDB ID: 3ET3) with FlexX score $-11.98,-9.69$ and -21.48 respectively followed by core hoping. Conclusion: Docking studies revealed that hydrogen-bonding interactions are crucial for the binding of ligands with the target. Core replacement of the best-docked conformations of the selected ligand is performed in order to obtain more potent and novel ligands.
\end{abstract}

Key words: Quantitative structure-activity relationship, Multiple Linear Regression, Molecular docking, Drug Likeness, Hydrogen-bonding interaction.

\section{INTRODUCTION}

Type 2 Diabetes Mellitus (T2DM) is an important public health problem, one of four priority Non-Communicable Diseases (NCDs) targeted for action by world leaders. Both the number of cases and the prevalence of diabetes have been steadily increasing over the past few decades. ${ }^{1,2}$ Worldwide, almost 0.422 billion grown-ups are existing with T2DM in 2014, as compared to 0.108 billion in $1980 .{ }^{3}$ The overall occurrence (age-standardized) of T2DM has approximately doubled since 1980 , escalating from $4.7 \%$ towards $8.5 \%$ in 2014 in the population of adult. ${ }^{4,5}$ India has been termed as the "Diabetes capital of the world" as it is lead- ing the world with the biggest number of diabetic subjects. ${ }^{6}$

There are currently various curative treatment available and therapy is reliant on the use of existing anti-diabetic drugs having their own long term toxic effects. ${ }^{7}$ Therefore it is crucial for identifying such molecules which are effective and possessing least side effects. Several authors have been studied PPAR as a potential target to treat these diseases. ${ }^{8}$ The Peroxisome Proliferator-Activated Receptors (PPARs) are categorized under the nuclear receptor superfamily are transcription factors activated by ligands. ${ }^{9} 10$ PPARs have three subtypes namely $\operatorname{PPAR} \alpha, \operatorname{PPAR} \beta / \delta$ and PPAR $\gamma$ and they divulge a usual impact
Submission Date: 18-02-2019; Revision Date: 17-05-2019; Accepted Date: 26-06-2019

DOI: 10.5530/ijper.53.3s.99 Correspondence: Ms. Neha Verma,

Research Scholar, Department of Mathematics, Bioinformatics and Computer Applications, MANIT, Bhopal-462001, Madhya Pradesh, INDIA. Phone: +91 8770381986 E-mail: nehav2314@gmail. com

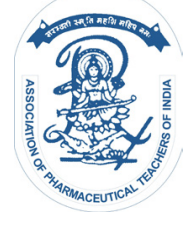

www.ijper.org 
on target cells as their actions are restricted to specific tissue types. ${ }^{11-13}$ All three receptors are important regulators in multiple physiological pathways, such as glucose homeostasis, fatty acid metabolism, inflammation and cellular differentiation. ${ }^{14,15}$ Activation of PPARs improves the condition of insulin resistance and therefore PPARs became a primary target in the treatment of type 2 diabetes. ${ }^{16}$ Derivatives of indanyl acetic acid are synthetic ligands which exhibit unique PPAR agonistic activities. ${ }^{17}$ Structure and ligand-based approaches have been successfully employed in the development of new drugs. ${ }^{18}$

The computational methods in drug discovery collectively termed pharmacoinformatics includes StructureActivity Relationship, ${ }^{19}$ virtual screening, ${ }^{20}$ molecular docking $^{21}$ and ADMET prediction ${ }^{22}$ have proven their pivotal role in the pharmaceutical industry for lead identification and optimization. Several research groups worldwide identified PPAR agonists using pharmacoinformatics approaches for potential application for the treatment of T2DM. In the present study, these pharmacoinformatic techniques are used to identify important features necessary for a compound to behave as an activator of PPAR- $\alpha$, PPAR $-\gamma$ and PPAR $-\delta / \beta$ receptors. ${ }^{10,23}$

\section{MATERIALS AND METHODS Dataset}

A set comprising of 69 indanyl acetic acid compounds bearing substituted phenyl tail groups, 4-heteroarylsubstituted aryl tail groups and thiazolylphenyl groups is taken from the available literature and used in the present study. ${ }^{17}$ The selected compounds for the data set shared the same assay i.e. FRET assay procedure with significant variations in their structures and potency profiles. The half-maximal effective concentration of the compounds included in data set, with $\mathrm{EC}_{50}$ values varying from 47 to $10000 \mathrm{nM}, 0.58$ to $2230 \mathrm{nM}$ and 12 to $10000 \mathrm{nM}$ for $\operatorname{PPAR} \alpha, \delta$ and $\gamma$ subtypes respectively which are converted into molar values. These are then converted into $\mathrm{pEC}_{50}$ values using the formula given below.

$$
\mathrm{pEC}_{50}=-\log 10\left[\mathrm{EC}_{50}\right]
$$

The structures of ligands are generated using the Chem Draw Ultra 7.0 software package. ${ }^{24}$ The energy minimization is performed for each ligand using the Chem3Dultra software. The X-ray crystal structure of the PPAR receptor is obtained from the Protein Data Bank (PDB ID: 3ET1, 3ET2 and 3ET3). ${ }^{25,26}$ The active site coordinates of the co-crystallized ligand are used for molecular docking studies to calculate the docking scores using FlexX algorithms. ${ }^{27}$ The FlexX program is used for docking of the receptor with the ligands qualifying all the drug-likeness filters like Lipinski's rule, Ghose filter, veber rules as well as a Quantitative Estimate of Drug-likeness (QED) calculated using DruLiTo software. ${ }^{28}$

\section{Multiple Linear Regression}

MLR analysis is a method for establishing the relationship between a single dependent variable and collection of independent variables (or predictors). ${ }^{29,30}$ It relies on the assumption that the variation in molecular properties of compounds can be related to changes in their structural/physicochemical properties. ${ }^{31}$ The optimized structures are used for all subsequent calculations. The $\mathrm{EC}_{50}$ data on indanyl acetic acid derivatives (Table 1) are grabbed from the literature. ${ }^{17}$ the dataset is randomly divided into a training set for creating QSAR models and a test set for the validation of the excellence of the models. All compounds in the test set contain the Biological Activity (BA) within the maximum and minimum value range of the $\mathrm{BA}$ of training set compounds.

\section{Physicochemical properties of Active Compounds}

Bad pharmacokinetic properties are one of the major cause for cessation of the generation of drug candidates. The drug detrition is a significant issue at clinical stages of drug development due to insufficient pharmacokinetics and pharmacodynamics examination. Numerous physicochemical properties like drug-like properties and toxicity of all the compounds in the dataset are evaluated employing the open source tools such as DruLiTo. ${ }^{28}$

\section{Molecular Docking}

The three-dimensional crystal structures of three selected molecular targets involved in the regulation of glucose homeostasis are obtained from PDB. ${ }^{25}$ The receptors are prepared by removal of heteroatoms such as water, ions and addition of polar hydrogens using the FlexX software. ${ }^{27}$ The active site is defined as including all atoms within a $6.5 \mathrm{~A}^{\circ}$ radius of the co-crystallized ligand. The docking scores (FlexX-Score) of the ligands are computed from the FlexX docked ligand-receptor complexes. Docking studies are performed for 100 generations and the energetically favorable conformations are analyzed. One complex structure for every ligand is selected as the best fit based on the orientations of ligand and its score is added to the molecular spreadsheet.

\section{Fragment-Based Drug Design}

The ReCore module developed by BioSolveIT is a useful suite for Fragment-Based Drug Design (FBDD). ${ }^{32}$ 


\begin{tabular}{|c|c|c|c|c|c|c|c|}
\hline \multirow[t]{2}{*}{ S.No. } & \multirow[t]{2}{*}{ Structure } & \multicolumn{2}{|c|}{ PPAR- $\alpha$} & \multicolumn{2}{|c|}{ PPAR-ס } & \multicolumn{2}{|c|}{ PPAR-Y } \\
\hline & & Actual & Predicted & Actual & Predicted & Actual & Predicted \\
\hline C1 & & 5.00 & 4.91 & 7.36 & 7.98 & 5.00 & 5.56 \\
\hline C2 & & 5.70 & 5.28 & 8.05 & 8.34 & 5.21 & 5.53 \\
\hline C3 & & $5.79^{*}$ & 5.31 & 8.38 & 8.37 & 5.25 & 5.41 \\
\hline C4 & & 5.17 & 4.84 & 7.89 & 8.45 & 5.99 & 5.74 \\
\hline C5 & & 5.00 & 5.01 & 8.17 & 8.14 & 5.52 & 5.62 \\
\hline C6 & & 5.00 & 5.30 & $8.18^{*}$ & 8.18 & 6.03 & 5.70 \\
\hline C7 & & 5.24 & 4.99 & 8.14 & 7.65 & $6.20^{*}$ & 5.50 \\
\hline C8 & & 5.00 & 5.01 & 7.25 & 7.94 & 5.10 & 5.27 \\
\hline C9 & & 5.00 & 5.13 & 7.17 & 7.73 & 5.00 & 5.45 \\
\hline C10 & & 5.03 & 5.13 & 8.34 & 7.90 & 5.66 & 5.77 \\
\hline C11 & & 5.00 & 5.49 & 8.62 & 8.09 & 5.82 & 6.20 \\
\hline C12 & & 5.94 & 5.90 & 8.80 & 8.14 & $6.52^{*}$ & 6.63 \\
\hline C13 & & 5.40 & 5.58 & 7.60 & 7.79 & 6.24 & 6.34 \\
\hline C14 & & 5.00 & 5.12 & $8.12^{*}$ & 8.07 & 5.78 & 5.76 \\
\hline C15 & & 6.16 & 5.39 & 8.57 & 8.31 & 5.78 & 6.01 \\
\hline C16 & & 5.13 & 5.10 & 7.85 & 7.65 & 6.16 & 5.93 \\
\hline C17 & & 5.00 & 5.42 & 8.66 & 8.01 & 5.91 & 5.85 \\
\hline C18 & & 5.00 & 5.32 & 7.96 & 8.03 & $6.26^{*}$ & 6.30 \\
\hline C19 & & 5.04 & 4.88 & 6.44 & 6.81 & 5.25 & 5.84 \\
\hline $\mathrm{C} 20$ & & 5.16 & 5.20 & 5.65 & 5.68 & 5.06 & 5.94 \\
\hline C21 & & $6.28^{*}$ & 6.31 & 8.47 & 8.23 & 6.80 & 6.69 \\
\hline C22 & & 5.00 & 5.48 & $8.23^{*}$ & 8.61 & 6.19 & 6.33 \\
\hline $\mathrm{C} 23$ & & 5.49 & 5.32 & 8.42 & 8.61 & 5.39 & 5.90 \\
\hline C24 & & 5.18 & 5.05 & 8.82 & 8.71 & 6.13 & 6.00 \\
\hline
\end{tabular}




\begin{tabular}{|c|c|c|c|c|c|c|c|}
\hline \multirow[t]{2}{*}{ S.No. } & \multirow[t]{2}{*}{ Structure } & \multicolumn{2}{|c|}{ PPAR- $\alpha$} & \multicolumn{2}{|c|}{ PPAR-ס } & \multicolumn{2}{|c|}{ PPAR-Y } \\
\hline & & Actual & Predicted & Actual & Predicted & Actual & Predicted \\
\hline C25 & & 5.77 & 5.34 & 8.64 & 8.43 & $6.26^{*}$ & 6.10 \\
\hline C26 & & 5.00 & 5.45 & 7.37 & 8.32 & 6.31 & 6.47 \\
\hline C27 & & 5.14 & 5.33 & 7.96 & 8.46 & 6.01 & 6.12 \\
\hline C28 & & 5.00 & 5.13 & 8.25 & 8.07 & 6.52 & 6.69 \\
\hline C29 & & 5.28 & 5.47 & 8.66 & 8.62 & 6.26 & 6.12 \\
\hline C30 & & 5.54 & 5.75 & 9.02 & 8.86 & 5.75 & 6.23 \\
\hline C31 & & 6.96 & 6.45 & $8.80^{*}$ & 8.25 & 7.32 & 7.05 \\
\hline C32 & & 6.59 & 5.97 & 9.24 & 8.38 & $6.43^{*}$ & 6.55 \\
\hline C33 & & 6.57 & 6.09 & 8.57 & 8.43 & 6.02 & 6.57 \\
\hline C34 & & $5.89^{*}$ & 5.98 & 8.36 & 8.50 & 6.06 & 6.13 \\
\hline C35 & & 6.83 & 6.63 & 7.96 & 8.43 & 7.35 & 7.17 \\
\hline C36 & & 6.60 & 6.35 & 8.60 & 8.39 & 7.19 & 6.80 \\
\hline C37 & & 7.06 & 6.92 & 7.96 & 8.37 & 7.75 & 7.74 \\
\hline C38 & & 6.71 & 6.87 & 8.48 & 8.52 & 7.26 & 7.41 \\
\hline C39 & & 6.36 & 6.39 & $8.54^{*}$ & 8.68 & $6.48^{*}$ & 6.70 \\
\hline C40 & & 5.90 & 6.58 & 8.54 & 8.62 & 6.55 & 6.83 \\
\hline C41 & & 7.08 & 6.68 & 8.40 & 8.56 & 7.48 & 7.31 \\
\hline $\mathrm{C} 42$ & & 6.95 & 6.60 & 8.89 & 8.73 & 5.92 & 6.82 \\
\hline C43 & & 6.48 & 7.18 & 8.46 & 8.51 & 7.03 & 7.75 \\
\hline C44 & & $7.28^{*}$ & 6.71 & 8.39 & 8.68 & 6.94 & 7.28 \\
\hline C45 & & 6.41 & 6.80 & $8.66^{*}$ & 8.75 & 6.53 & 7.10 \\
\hline C46 & & 7.37 & 7.39 & 8.72 & 8.55 & $7.92^{*}$ & 8.00 \\
\hline C47 & & 7.36 & 6.91 & 8.48 & 8.72 & 7.08 & 7.52 \\
\hline C48 & & 7.00 & 7.21 & 8.40 & 8.65 & 7.38 & 7.63 \\
\hline C49 & $x_{0} \times 1.0$ & 6.48 & 6.73 & 8.89 & 8.89 & 6.74 & 6.83 \\
\hline C50 & 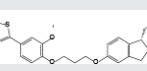 & 6.28 & 6.21 & 8.28 & 8.67 & 6.67 & 6.87 \\
\hline
\end{tabular}




\begin{tabular}{|c|c|c|c|c|c|c|c|}
\hline \multirow[t]{2}{*}{ S.No. } & \multirow[t]{2}{*}{ Structure } & \multicolumn{2}{|c|}{ PPAR- $\alpha$} & \multicolumn{2}{|c|}{ PPAR- $\delta$} & \multicolumn{2}{|c|}{ PPAR-Y } \\
\hline & & Actual & Predicted & Actual & Predicted & Actual & Predicted \\
\hline C51 & & 5.60 & 6.16 & 8.16 & 8.22 & 6.32 & 6.72 \\
\hline C52 & & 6.76 & 6.75 & 7.75 & 8.12 & 7.77 & 7.80 \\
\hline C53 & & 6.25 & 6.27 & 8.47 & 8.13 & $7.31^{*}$ & 7.02 \\
\hline C54 & & $6.85^{\star}$ & 6.85 & 8.36 & 8.09 & 7.70 & 8.12 \\
\hline C55 & & 5.00 & 5.04 & $6.66^{*}$ & 7.97 & 6.51 & 5.86 \\
\hline C56 & & 5.77 & 5.62 & 7.34 & 7.67 & 6.62 & 7.00 \\
\hline C57 & & 5.00 & 5.14 & 7.55 & 7.88 & 6.00 & 6.31 \\
\hline C58 & & 5.19 & 5.19 & 7.92 & 7.65 & 6.05 & 6.34 \\
\hline C59 & & 5.61 & 5.74 & 8.34 & 8.20 & $7.57^{*}$ & 7.09 \\
\hline $\mathrm{C} 60$ & & 5.24 & 5.97 & 8.89 & 8.64 & 6.52 & 6.47 \\
\hline C61 & & 6.12 & 6.08 & 8.52 & 8.41 & 7.16 & 6.84 \\
\hline C62 & & 7.00 & 6.27 & 8.92 & 8.54 & 6.12 & 6.46 \\
\hline $\mathrm{C} 63$ & & $6.98^{*}$ & 6.86 & $8.70^{*}$ & 8.30 & 7.39 & 7.45 \\
\hline C64 & & 5.87 & 6.38 & 8.47 & 8.55 & 6.04 & 6.80 \\
\hline $\mathrm{C} 65$ & & 7.33 & 6.96 & 8.64 & 8.48 & 7.35 & 7.71 \\
\hline C66 & & 6.75 & 6.48 & 8.52 & 8.52 & $7.36^{*}$ & 7.02 \\
\hline C67 & & 7.22 & 6.92 & 8.50 & 8.28 & 7.47 & 7.55 \\
\hline C68 & & 6.82 & 6.44 & 8.22 & 8.46 & 6.75 & 6.90 \\
\hline C69 & & 6.28 & 6.71 & 8.27 & 8.50 & 6.44 & 7.13 \\
\hline
\end{tabular}

*test set compounds

This module alters hit ligands by replacing their core. 3D Fragments are generated to replace the core using a vector-based scheme and the resulting structures are scored using the FlexX docking program. ${ }^{33,34}$ Recore offers constructive hopping of scaffold, replacement of $3 \mathrm{D}$ core and linking and merging of the fragment. ReCore delivers back a rank-sorted list of fragments within seconds (depending on the complexity of the query). The hit fragments are sorted according to how well they comply with the query using a multidimensional Euclidean distance.

\section{RESULTS AND DISCUSSION}

The substituted indanyl acetic acid derivatives reportedly exhibit strong agonistic activities against PPARs. In the current study, we examined only the agonistic activities 
against PPARs using 2DQSAR studies, specifically MLR and molecular docking scores.

\section{MLR Analysis}

The MLR models are derived for a dataset of 69 PPAR activators. The statistical parameters associated with the MLR analysis of PPAR $\alpha, \operatorname{PPAR} \beta / \delta$ and PPAR $\gamma$, represented by respective model I, II and III are listed in Table 2 . The prediction ability of models is determined using a set of test compounds not included in the model generation. The values of experimental and predicted activities of all models are depicted in Table 1. The graphs of actual activity versus predicted activity of the training set and test set for all models are illustrated in Figure 1.

$$
\begin{aligned}
\mathrm{pEC}_{50}= & 3.439+1.024 \times \text { chi1 } \mathrm{v}-.520 \times \\
& \text { a_don- } .661 \times \mathrm{SMR}-.015 \times \text { PEOE_VSA2_A }
\end{aligned}
$$

Model 1 represents the MLR equation for PPAR- $\alpha$ agonists which shows chi1v as the important feature contributing positively to the activity. The a_don (Number of hydrogen bond donor atoms) and SMR (Molecular refractivity) descriptors dominate in explaining the variation in activity as evidenced by the final QSAR equation. The model also reveals the importance of PEOE_VSA2 (Partial Equalization of Orbital Electronegativity) descriptor contributing towards the BA.

$$
\begin{aligned}
\mathrm{pEC}_{50}= & -2.770-2.014 \times \mathrm{a} \_\mathrm{nCl}+0.233 \times \\
& \text { dipoleY }-0.031 \times \text { vsa_pol }+2.157 \times \\
& \text { VDistEq }-16.323 \times \mathrm{GCUT} \text { SMR_1 }
\end{aligned}
$$

Model 2 represents the MLR equation for PPAR- $\delta$ agonists in which negative value of the coefficients of a_ncl (Number of chlorine atoms), vsa_pol (Approximation to the sum of VDW surface areas $\left(\AA^{2}\right)$ of polar atoms) and GCUT_SMR_1 (atomic contribution to molar refractivity) descriptors reveals that increase in a_ncl, vas_pol and GCUT_SMR_1 value also increases the activity of the molecule. A positive value of the

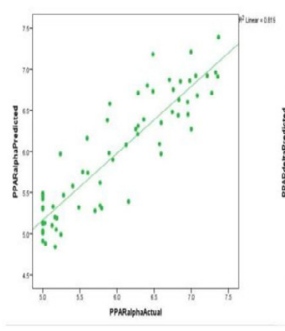

(A)

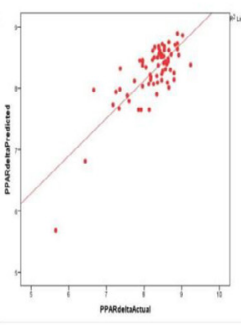

(B)

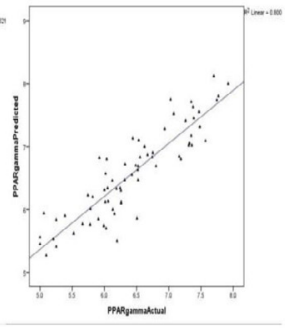

(C)
Figure 1: Scatter Plot between Actual and Predicted Activity (pEC50) of (A) PPAR- $\alpha$ (B) PPAR- $\delta$ and (C) PPAR-y agonists dipole (coefficient of dipole) and VDistEq (sum of the distance matrix entries) indicates that it has a positive impact on the biological activity of the ligands.

$$
\begin{aligned}
\mathrm{pEC}_{50}= & -373455.688+.013 \times \mathrm{vol}-0.636 \times \\
& \text { lip_don }+127961.965 \times \text { BCUT_SMR_3 }-10.619 \times \\
& \text { BCUT_SLOGP_1 }-.009 \times \text { PEOE_VSA1 }
\end{aligned}
$$

Model 3 represents the MLR equation for PPAR- $\gamma$ agonists which reveals that vol (van der Waals volume) and BCUT_SMR_3 (atomic contribution to molar refractivity) descriptors positively affects the activity of ligands while the negative value of lip_don (The number of $\mathrm{OH}$ and $\mathrm{NH}$ atoms) and BCUT_SLOGP_1 (atomic contribution to $\log \mathrm{P})$ descriptor indicates that increase in lip_don and BCUT_SLOGP_1 is responsible for an increase in the activity of the molecule.

\begin{tabular}{|c|c|c|c|}
\hline & $\begin{array}{l}\text { PPARa I } \\
\text { Model } 1\end{array}$ & $\begin{array}{l}\text { PPARס / } \\
\text { Model } 2\end{array}$ & $\begin{array}{l}\text { PPARy I } \\
\text { Model } 3\end{array}$ \\
\hline \multicolumn{4}{|c|}{ MLR Analysis } \\
\hline $\begin{array}{l}n \operatorname{Tr} \text { (No. of comp. in } \\
\text { training set) }\end{array}$ & 63 & 59 & 61 \\
\hline $\begin{array}{l}n T s \text { (No. of comp. in } \\
\text { the test set) }\end{array}$ & 6 & 10 & 8 \\
\hline $\begin{array}{l}R^{2} \text { (Correlation } \\
\text { Coefficient) }\end{array}$ & 0.81 & 0.66 & 0.82 \\
\hline $\begin{array}{c}R^{2} \text { pred(Predicted } \\
\text { Correlation Coefficient) }\end{array}$ & 0.86 & 0.90 & 0.58 \\
\hline $\begin{array}{l}\text { Q2 (Cross validated } \\
\text { Correlation Coefficient) }\end{array}$ & 0.81 & 0.66 & 0.77 \\
\hline $\begin{array}{c}\text { See (Standard Error of } \\
\text { Estimation) }\end{array}$ & 0.35 & 0.36 & 0.34 \\
\hline$S p$ & 0.32 & 0.17 & 0.15 \\
\hline \multicolumn{4}{|c|}{ Docking Analysis (FlexX Score) } \\
\hline C5 & -11.16 & -17.93 & -12.08 \\
\hline $\mathrm{C7}$ & -12.17 & -12.81 & -18.86 \\
\hline C16 & -11.98 & -9.69 & -21.48 \\
\hline C18 & -9.65 & -15.61 & -15.07 \\
\hline Natural Ligand & -19.83 & -22.54 & -23.92 \\
\hline $\begin{array}{c}\text { Zinc03584559 } \\
\text { (Obtained from } \\
\text { Recore) }\end{array}$ & -21.76 & -18.51 & -31.09 \\
\hline
\end{tabular}

\section{ADMET Prediction}

Distinct pharmacokinetic characteristics of the compounds considered for the study are subjected to ADME predictions by DruLiTo. The compounds are assessed for their fundamental parameters of Lipinski's rule of 5 and furthermore various pharmacokinetic properties. ${ }^{35}$ Table 3 shows the results obtained from 


\begin{tabular}{|c|c|c|c|c|c|c|c|c|c|c|c|c|c|}
\hline Sr. No. & MW & $\log p$ & Alogp & HBA & HBD & TPSA & AMR & $\mathrm{nRB}$ & nAtom & $\begin{array}{l}\text { nAcidic } \\
\text { Group }\end{array}$ & $R C$ & nRigidB & nArom \\
\hline $\mathrm{C} 1$ & 304.99 & 5.35 & -0.77 & 2.00 & 0.00 & 35.53 & 39.46 & 8.00 & 25.00 & 0.00 & 3.00 & 18.00 & 2.00 \\
\hline $\mathrm{C} 2$ & 328.99 & 6.24 & -0.46 & 2.00 & 0.00 & 35.53 & 49.81 & 9.00 & 27.00 & 0.00 & 3.00 & 19.00 & 2.00 \\
\hline C3 & 373.98 & 6.26 & 0.33 & 2.00 & 0.00 & 35.53 & 45.90 & 9.00 & 29.00 & 0.00 & 3.00 & 21.00 & 2.00 \\
\hline C4 & 389.98 & 5.95 & 1.32 & 2.00 & 0.00 & 44.76 & 47.89 & 10.00 & 30.00 & 0.00 & 3.00 & 21.00 & 2.00 \\
\hline C5 & 332.98 & 4.70 & -0.82 & 2.00 & 0.00 & 44.76 & 46.78 & 9.00 & 27.00 & 0.00 & 3.00 & 19.00 & 2.00 \\
\hline C6 & 344.98 & 5.12 & -0.57 & 2.00 & 0.00 & 44.76 & 51.07 & 10.00 & 28.00 & 0.00 & 3.00 & 19.00 & 2.00 \\
\hline $\mathrm{C} 7$ & 330.99 & 4.49 & -0.73 & 3.00 & 0.00 & 59.32 & 45.66 & 8.00 & 27.00 & 0.00 & 3.00 & 20.00 & 2.00 \\
\hline $\mathrm{C} 8$ & 316.99 & 5.68 & -0.12 & 2.00 & 0.00 & 35.53 & 44.96 & 8.00 & 26.00 & 0.00 & 3.00 & 19.00 & 2.00 \\
\hline $\mathrm{C9}$ & 328.99 & 6.00 & 0.52 & 2.00 & 0.00 & 35.53 & 50.47 & 8.00 & 27.00 & 0.00 & 3.00 & 20.00 & 2.00 \\
\hline C10 & 328.99 & 5.79 & 0.52 & 2.00 & 0.00 & 35.53 & 50.47 & 8.00 & 27.00 & 0.00 & 3.00 & 20.00 & 2.00 \\
\hline C11 & 340.99 & 6.60 & -0.94 & 2.00 & 0.00 & 35.53 & 53.14 & 10.00 & 28.00 & 0.00 & 3.00 & 19.00 & 2.00 \\
\hline C12 & 409.98 & 7.51 & 0.16 & 2.00 & 0.00 & 35.53 & 59.57 & 11.00 & 32.00 & 0.00 & 3.00 & 22.00 & 2.00 \\
\hline C13 & 366.99 & 5.74 & -0.91 & 3.00 & 0.00 & 59.32 & 59.34 & 10.00 & 30.00 & 0.00 & 3.00 & 21.00 & 2.00 \\
\hline C14 & 344.98 & 5.02 & -0.18 & 2.00 & 0.00 & 44.76 & 52.28 & 9.00 & 28.00 & 0.00 & 3.00 & 20.00 & 2.00 \\
\hline C15 & 356.98 & 5.59 & -0.51 & 2.00 & 0.00 & 44.76 & 57.13 & 10.00 & 29.00 & 0.00 & 3.00 & 20.00 & 2.00 \\
\hline C16 & 358.99 & 4.05 & -0.78 & 3.00 & 0.00 & 68.55 & 52.98 & 9.00 & 29.00 & 0.00 & 3.00 & 21.00 & 2.00 \\
\hline C17 & 356.98 & 5.44 & 0.08 & 2.00 & 0.00 & 44.76 & 56.57 & 10.00 & 29.00 & 0.00 & 3.00 & 20.00 & 2.00 \\
\hline C18 & 371.00 & 4.41 & -0.75 & 5.00 & 0.00 & 66.24 & 42.36 & 9.00 & 30.00 & 0.00 & 4.00 & 23.00 & 3.00 \\
\hline C19 & 424.99 & 3.65 & -1.38 & 7.00 & 0.00 & 83.31 & 55.23 & 11.00 & 34.00 & 0.00 & 4.00 & 25.00 & 3.00 \\
\hline $\mathrm{C} 20$ & 405.97 & 4.90 & -0.01 & 5.00 & 0.00 & 66.24 & 47.98 & 9.00 & 31.00 & 0.00 & 4.00 & 24.00 & 3.00 \\
\hline $\mathrm{C} 21$ & 469.96 & 6.60 & 1.57 & 4.00 & 0.00 & 89.55 & 59.19 & 10.00 & 35.00 & 0.00 & 4.00 & 27.00 & 3.00 \\
\hline $\mathrm{C} 22$ & 384.96 & 6.43 & -0.18 & 2.00 & 0.00 & 63.77 & 47.26 & 9.00 & 30.00 & 0.00 & 4.00 & 23.00 & 3.00 \\
\hline $\mathrm{C} 23$ & 368.98 & 5.33 & -0.92 & 2.00 & 0.00 & 48.67 & 41.08 & 9.00 & 30.00 & 0.00 & 4.00 & 23.00 & 3.00 \\
\hline C24 & 416.00 & 6.97 & -1.19 & 3.00 & 1.00 & 51.32 & 42.76 & 9.00 & 35.00 & 0.00 & 5.00 & 28.00 & 4.00 \\
\hline $\mathrm{C} 25$ & 378.99 & 5.60 & -1.19 & 3.00 & 0.00 & 48.42 & 42.76 & 9.00 & 31.00 & 0.00 & 4.00 & 24.00 & 3.00 \\
\hline $\mathrm{C} 26$ & 406.99 & 5.15 & -1.24 & 3.00 & 0.00 & 57.65 & 50.08 & 10.00 & 33.00 & 0.00 & 4.00 & 25.00 & 3.00 \\
\hline $\mathrm{C} 27$ & 380.99 & 4.78 & -0.77 & 4.00 & 0.00 & 61.31 & 39.46 & 9.00 & 31.00 & 0.00 & 4.00 & 24.00 & 3.00 \\
\hline $\mathrm{C} 28$ & 448.98 & 5.62 & -0.87 & 4.00 & 0.00 & 79.77 & 54.09 & 11.00 & 36.00 & 0.00 & 4.00 & 26.00 & 3.00 \\
\hline $\mathrm{C} 29$ & 390.99 & 6.48 & -0.75 & 3.00 & 0.00 & 48.42 & 48.50 & 9.00 & 32.00 & 0.00 & 4.00 & 25.00 & 3.00 \\
\hline C30 & 447.99 & 7.10 & -0.09 & 3.00 & 0.00 & 48.42 & 49.20 & 10.00 & 35.00 & 0.00 & 4.00 & 27.00 & 3.00 \\
\hline C31 & 422.96 & 6.67 & -0.35 & 3.00 & 0.00 & 76.66 & 60.93 & 11.00 & 33.00 & 0.00 & 4.00 & 24.00 & 3.00 \\
\hline C32 & 414.96 & 4.97 & -0.23 & 3.00 & 0.00 & 85.89 & 54.57 & 10.00 & 32.00 & 0.00 & 4.00 & 24.00 & 3.00 \\
\hline C33 & 426.96 & 5.47 & 0.21 & 3.00 & 0.00 & 85.89 & 60.30 & 10.00 & 33.00 & 0.00 & 4.00 & 25.00 & 3.00 \\
\hline C34 & 398.96 & 5.91 & 0.26 & 3.00 & 0.00 & 76.66 & 52.99 & 9.00 & 31.00 & 0.00 & 4.00 & 24.00 & 3.00 \\
\hline C35 & 434.96 & 6.95 & -0.11 & 3.00 & 0.00 & 76.66 & 66.42 & 11.00 & 34.00 & 0.00 & 4.00 & 25.00 & 3.00 \\
\hline C36 & 438.96 & 5.83 & -0.46 & 3.00 & 0.00 & 85.89 & 63.38 & 11.00 & 34.00 & 0.00 & 4.00 & 25.00 & 3.00 \\
\hline C37 & 470.96 & 8.21 & 1.21 & 3.00 & 0.00 & 76.66 & 80.06 & 12.00 & 37.00 & 0.00 & 4.00 & 27.00 & 3.00 \\
\hline C38 & 491.96 & 8.17 & 0.75 & 3.00 & 0.00 & 76.66 & 67.37 & 12.00 & 37.00 & 0.00 & 4.00 & 27.00 & 3.00 \\
\hline C39 & 483.95 & 6.47 & 0.87 & 3.00 & 0.00 & 85.89 & 61.01 & 11.00 & 36.00 & 0.00 & 4.00 & 27.00 & 3.00 \\
\hline C40 & 434.96 & 6.74 & -0.65 & 3.00 & 0.00 & 76.66 & 64.88 & 11.00 & 34.00 & 0.00 & 4.00 & 25.00 & 3.00 \\
\hline C41 & 462.96 & 6.30 & -0.70 & 3.00 & 0.00 & 85.89 & 72.19 & 12.00 & 36.00 & 0.00 & 4.00 & 26.00 & 3.00 \\
\hline C42 & 422.96 & 6.04 & 0.05 & 3.00 & 0.00 & 76.66 & 59.07 & 9.00 & 33.00 & 0.00 & 5.00 & 27.00 & 3.00 \\
\hline C43 & 458.96 & 7.29 & -0.13 & 3.00 & 0.00 & 76.66 & 72.75 & 11.00 & 36.00 & 0.00 & 5.00 & 28.00 & 3.00 \\
\hline $\mathrm{C} 44$ & 450.96 & 5.60 & 0.00 & 3.00 & 0.00 & 85.89 & 66.39 & 10.00 & 35.00 & 0.00 & 5.00 & 28.00 & 3.00 \\
\hline C45 & 450.96 & 5.60 & 0.00 & 3.00 & 0.00 & 85.89 & 66.39 & 10.00 & 35.00 & 0.00 & 5.00 & 28.00 & 3.00 \\
\hline
\end{tabular}




\begin{tabular}{|c|c|c|c|c|c|c|c|c|c|c|c|c|c|}
\hline Sr. No. & MW & $\log p$ & Alogp & HBA & HBD & TPSA & AMR & $n R B$ & nAtom & $\begin{array}{l}\text { nAcidic } \\
\text { Group }\end{array}$ & RC & nRigidB & nArom \\
\hline C46 & 470.96 & 7.65 & -0.42 & 3.00 & 0.00 & 76.66 & 75.66 & 11.00 & 37.00 & 0.00 & 5.00 & 29.00 & 3.00 \\
\hline $\mathrm{C} 47$ & 462.96 & 5.96 & -0.29 & 3.00 & 0.00 & 85.89 & 69.30 & 10.00 & 36.00 & 0.00 & 5.00 & 29.00 & 3.00 \\
\hline C48 & 474.96 & 6.92 & -0.53 & 3.00 & 0.00 & 85.89 & 74.63 & 11.00 & 37.00 & 0.00 & 5.00 & 29.00 & 3.00 \\
\hline C49 & 466.95 & 5.22 & -0.40 & 3.00 & 0.00 & 95.12 & 68.27 & 10.00 & 36.00 & 0.00 & 5.00 & 29.00 & 3.00 \\
\hline C50 & 462.96 & 6.39 & -0.23 & 3.00 & 0.00 & 85.89 & 54.57 & 10.00 & 36.00 & 0.00 & 5.00 & 29.00 & 4.00 \\
\hline C51 & 438.96 & 5.42 & 0.01 & 4.00 & 0.00 & 93.73 & 63.76 & 10.00 & 34.00 & 0.00 & 4.00 & 26.00 & 3.00 \\
\hline C52 & 474.96 & 6.67 & -0.16 & 4.00 & 0.00 & 93.73 & 77.43 & 12.00 & 37.00 & 0.00 & 4.00 & 27.00 & 3.00 \\
\hline C53 & 466.95 & 4.97 & -0.04 & 4.00 & 0.00 & 102.90 & 71.07 & 11.00 & 36.00 & 0.00 & 4.00 & 27.00 & 3.00 \\
\hline C54 & 500.96 & 6.33 & -0.34 & 5.00 & 0.00 & 96.97 & 85.50 & 13.00 & 39.00 & 0.00 & 4.00 & 28.00 & 3.00 \\
\hline C55 & 442.95 & 5.15 & 0.02 & 5.00 & 0.00 & 93.73 & 60.21 & 10.00 & 34.00 & 0.00 & 4.00 & 26.00 & 3.00 \\
\hline C56 & 478.95 & 6.40 & -0.15 & 5.00 & 0.00 & 93.73 & 73.88 & 12.00 & 37.00 & 0.00 & 4.00 & 27.00 & 3.00 \\
\hline C57 & 470.95 & 4.71 & -0.03 & 5.00 & 0.00 & 102.90 & 67.52 & 11.00 & 36.00 & 0.00 & 4.00 & 27.00 & 3.00 \\
\hline C58 & 494.95 & 5.27 & -1.04 & 6.00 & 0.00 & 93.73 & 75.43 & 13.00 & 38.00 & 0.00 & 4.00 & 27.00 & 3.00 \\
\hline C59 & 478.95 & 6.28 & -0.56 & 5.00 & 0.00 & 93.73 & 72.70 & 13.00 & 37.00 & 0.00 & 4.00 & 26.00 & 3.00 \\
\hline C60 & 414.96 & 5.47 & -0.23 & 3.00 & 0.00 & 85.89 & 54.57 & 10.00 & 32.00 & 0.00 & 4.00 & 24.00 & 3.00 \\
\hline C61 & 442.95 & 5.03 & -0.28 & 3.00 & 0.00 & 95.12 & 61.89 & 11.00 & 34.00 & 0.00 & 4.00 & 25.00 & 3.00 \\
\hline C62 & 426.96 & 5.90 & 0.02 & 3.00 & 0.00 & 85.89 & 58.87 & 11.00 & 33.00 & 0.00 & 4.00 & 24.00 & 3.00 \\
\hline C63 & 462.96 & 7.15 & -0.15 & 3.00 & 0.00 & 85.89 & 72.54 & 13.00 & 36.00 & 0.00 & 4.00 & 25.00 & 3.00 \\
\hline C64 & 454.95 & 5.45 & -0.03 & 3.00 & 0.00 & 95.12 & 66.18 & 12.00 & 35.00 & 0.00 & 4.00 & 25.00 & 3.00 \\
\hline C65 & 474.96 & 7.61 & -0.45 & 3.00 & 0.00 & 85.89 & 77.75 & 13.00 & 37.00 & 0.00 & 4.00 & 26.00 & 3.00 \\
\hline C66 & 466.95 & 5.91 & -0.33 & 3.00 & 0.00 & 95.12 & 71.39 & 12.00 & 36.00 & 0.00 & 4.00 & 26.00 & 3.00 \\
\hline C67 & 474.96 & 7.26 & 0.29 & 3.00 & 0.00 & 85.89 & 78.27 & 13.00 & 37.00 & 0.00 & 4.00 & 26.00 & 3.00 \\
\hline C68 & 466.95 & 5.56 & 0.41 & 3.00 & 0.00 & 95.12 & 71.91 & 12.00 & 36.00 & 0.00 & 4.00 & 26.00 & 3.00 \\
\hline C69 & 478.95 & 5.92 & -0.27 & 3.00 & 0.00 & 95.12 & 74.99 & 13.00 & 37.00 & 0.00 & 4.00 & 26.00 & 3.00 \\
\hline ZINC03584559 & 441.10 & 3.18 & -0.40 & 6.00 & 1.00 & 91.59 & 82.38 & 5.00 & 49.00 & 0.00 & 5.00 & 30.00 & 2.00 \\
\hline
\end{tabular}

DruLiTo with their permissible range. In general, an orally dynamic compound ought not to have more than 2 infringement of the Lipinski rule. The dynamic test compounds in the present study are not found disregarding the most extreme permissible limits of Lipinski rule and in this way demonstrating their drug likeliness properties. The ideal estimations of the descriptors, rotatable bonds and polar surface area likewise have an extraordinary impact on the oral bioavailability of the drug atoms. The important parameters with their permissible ranges are delineated in Table 3. The compounds C5, C7, C16 and C18 qualifying all the ADME filters are further used for docking Analysis.

\section{Molecular Docking}

PPAR $\alpha$ : The compounds qualifying all the ADME filters are further used for docking Analysis using FlexX software. All four compounds are docked for its binding interactions in the active site of (PDB 3ET1) protein. The binding pocket consisted of TYR464, TYR314, HIS440, PHE273, GLN277, CYS276, ILE354, SER280, etc. residues in the targeted protein as shown in Figure 2. For the interaction between ligands and receptor, the presence of a hydrogen bonding feature is found very crucial which is also observed in the QSAR studies. Residue TYR464 and TYR314 are found to show favorable interaction, including $\mathrm{HB}$ acceptor and $\mathrm{HB}$ donor with the best conformers. The TYR464 and TYR314 residues in receptor binding pocket interacts with the oxygen atom of the carboxyl group of conformers of compounds C5, C7, C16 and C18 with FlexX score $-11.16,-12.17,-11.98$ and -9.65 respectively.

PPAR $\delta:$ The minimum binding energy indicates that the PPAR $\delta$ receptor (PDB 3ET2) is successfully docked with indanyl acetic acid derivative is shown in Table 2. The possible binding modes of indanyl acetic acid derivative at PPAR $\delta$ active sites have been shown in Figure 2. PPAR $\delta$ protein residues TYR437, HIS287, HIS413, CYS249, PHE246, LEU433, etc. formed active site in the protein. Residue TYR437, HIS413 and HIS287 found to form H-bond with the ligand molecules. Ligand showed relatively good binding affinity as 


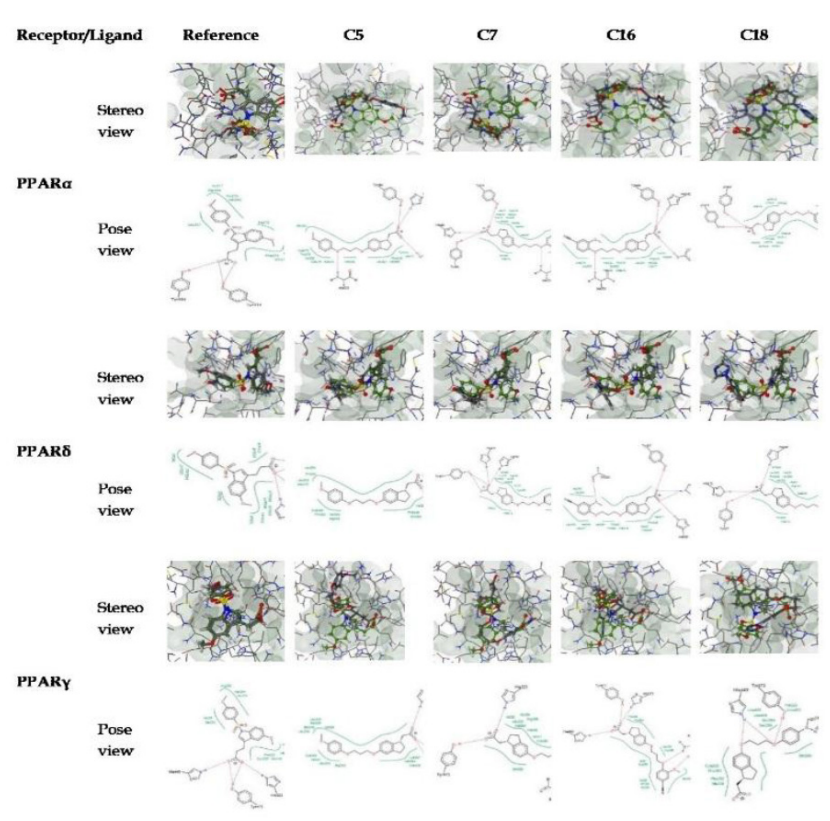

Figure 2: Molecular Docking between PPARa (PDB 3ET1), PPARס (PDB 3ET2) and PPARY (PDB 3ET3) receptors with natural ligand and Compound C5, C7, C16 and C18.

compared to the natural ligand as standard which showed minimum binding energy shown in Table 2.

PPAR $\gamma$ : To further observe the interactions, the compounds are docked for its binding interactions with the active site of (PDB 3ET3) protein. To calculate the docked scores of the agonist structures against PPAR $\gamma$, the molecular docking program FlexX is employed. In the current study, 100 conformational binding modes are generated for each ligand at the active site and the number of $\mathrm{HB}$ interactions are observed. The docked conformation of compounds revealed that the compound interacted with the binding pocket residues (TYR473, HIS449, HIS323, ARG288, CYS285, etc.) of receptor through several favorable interactions such as $\mathrm{HB}$ donor and acceptor with residue TYR473, HIS449 and HIS323 respectively as shown in Figure 2. The binding affinity of ligands is shown in Table 2.

\section{Fragment-Based Drug Design using Recore}

The ReCore module is used to modify the best docked hit C16 compounds by replacing their core. Fragments utilized to replace the chemical scaffold are generated in $3 \mathrm{D}$, to cut and replace the fragment, a vector based scheme is used. To score the resulting structures the FlexX docking program is used. The starting hit compound is altered with linker fragments that possess similar functional groups; The new fragment is grafted onto the starting fragment by overlapping. As a result, various compounds obtained out of which the bestscored compound is analyzed using FlexX. It is interesting

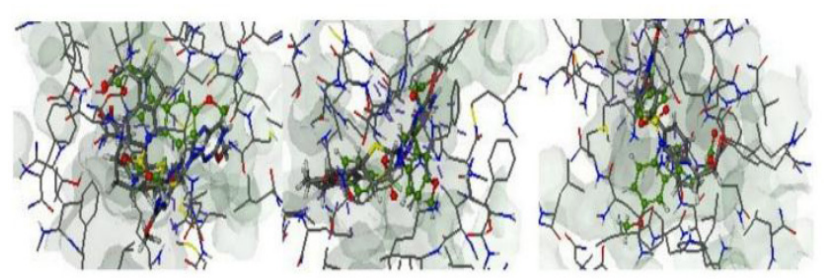

(A)

(B)

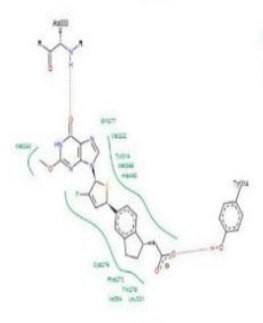

(a)

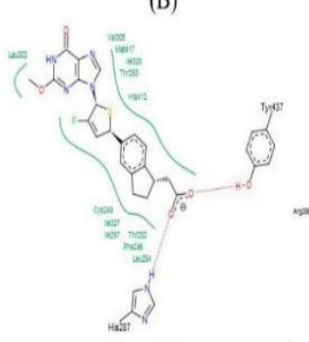

(b)
(C)

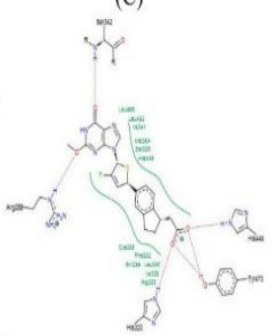

(c)
Figure 3: Molecular interaction between PPAR $\alpha$, PPARס and PPARy receptors with modified compound ZINC03584559 obtained from Recore analysis. A, B and C showing stereoview and $a, b$ and $c$ showing pose view of interaction between PPARa, PPARס and PPARy receptors with novel compound (modified ZINC03584559) respectively.

to note that compound containing ZINC03584559 ${ }^{36}$ in core exhibited better scores than the natural ligand and the other docked highly active compounds as shown in Table 2. The binding poses for the interaction are shown in Figure 3.

\section{CONCLUSION}

The combined computational approaches are applied to give insight into the structural basis and activation mechanism for a series of indanyl acetic acid derivatives as anti-diabetic agents. QSAR modeling is performed to provide a structural framework for understanding the structure-activity relationship of these compounds. The 2D-QSAR generated models exhibited good predictive power, correlation and satisfactory agreement between theory and experiment. Further, ADME predictions are performed for the set of compounds followed by molecular docking studies to generate possible binding poses for these compounds to PPARs. Conclusively, the hits obtained on virtual screening of the zinc database using RECORE module have provided new chemical starting points for design and development of novel PPAR targeting agents.

\section{ACKNOWLEDGEMENT}

Author Neha Verma would like to acknowledge the University Grant Commission for providing Rajiv Gandhi National Fellowship. 


\section{CONFLICT OF INTEREST}

The authors declare no conflict of interest.

\section{ABBREVIATIONS}

T2DM: Type 2 Diabetes Mellitus; NCD: Non-Communicable Diseases; PPAR: Peroxisome Proliferator-Activated Receptor; QED: Quantitative Estimate of Drug Likeness; QSAR: Quantitative Structure-Activity Relationship; MLR: Multiple Linear Regression; BA: Biological Activity; FBDD: Fragment-Based Drug Design; ADME: Absorption, Distribution, Metabolism and Excretion.

\section{REFERENCES}

1. DeFronzo RA. Pathogenesis of type 2 diabetes mellitus. Diabetes Epidemiology, Genetics, Pathogenesis, Diagnosis, Prevention and Treatment. 2018;181-253.

2. Short Term Effects of Type 2 Diabetes: HEALTH BASIS. 2017. Available from: http://mnbasis.com/short-term-effects-of-type-2-diabetes.html.

3. Organization WH. Global report on diabetes: World Health Organization. 2016.

4. Banner C, Göttlicher M, Widmark E, Sjövall J, Rafter J, Gustafsson JA. A systematic analytical chemistry/cell assay approach to isolate activators of orphan nuclear receptors from biological extracts: Characterization of peroxisome proliferator-activated receptor activators in plasma. Journal of Lipid Research. 1993;34(9):1583-91.

5. Guariguata L, Whiting DR, Hambleton I, Beagley J, Linnenkamp U, Shaw JE. Global estimates of diabetes prevalence for 2013 and projections for 2035. Diabetes Research and Clinical Practice. 2014;103(2):137-49.

6. Jamwal A, Kumar S. Antidiabetic Activity of Isolated Compound from Coccinia indica. Indian Journal of Pharmaceutical Education and Research. 2019;53(1):151-9.

7. Dodds S. The how-to for type 2: An overview of diagnosis and management of type 2 diabetes mellitus. Nursing Clinics. 2017;52(4):513-22.

8. Nandy A, Roy K, Saha A. A Comparative Study on Selective PPAR Modulators through Quantitative Structure-activity Relationship, Pharmacophore and Docking Analyses. Current Computer-aided Drug Design. 2018;14(1):54-67.

9. Carrieri A, Giudici M, Parente M, DeRosas M, Piemontese L, Fracchiolla G, et al. Molecular determinants for nuclear receptors selectivity: Chemometric analysis, dockings and site-directed mutagenesis of dual peroxisome proliferator-activated receptors $\alpha / y$ agonists. European Journal of Medicinal Chemistry. 2013;63:321-32.

10. Willson TM, Brown PJ, Sternbach DD, Henke BR. The PPARs: from orphan receptors to drug discovery. Journal of Medicinal Chemistry. 2000;43(4):527-50.

11. Braissant O, Foufelle F, Scotto C, Dauça M, Wahli W. Differential expression of Peroxisome Proliferator-Activated Receptors (PPARs): Tissue distribution of PPAR-alpha,-beta and-gamma in the adult rat. Endocrinology. 1996;137(1):354-66

12. Mukherjee R, Jow L, Croston GE, Paterniti JR. Identification, characterization and tissue distribution of human Peroxisome Proliferator-Activated Receptor (PPAR) isoforms PPARy2 versus PPARY1 and activation with retinoid $X$ receptor agonists and antagonists. Journal of Biological Chemistry. 1997;272(12):8071-6.

13. Mukherjee R, Jow L, Noonan D, McDonnell D. Human and rat peroxisome proliferator activated receptors (PPARs) demonstrate similar tissue distribution but different responsiveness to PPAR activators. The Journal of Steroid Biochemistry and Molecular Biology. 1994;51(3-4):157-66.
14. Kordik CP, Reitz AB. Pharmacological treatment of obesity: Therapeutic strategies. Journal of Medicinal Chemistry. 1999;42(2):181-201.

15. Lin Q, Ruuska SE, Shaw NS, Dong D, Noy N. Ligand selectivity of the peroxisome proliferator-activated receptor $\alpha$. Biochemistry. 1999;38(1):185-90.

16. Auwerx J. PPARy, the ultimate thrifty gene. Diabetologia. 1999;42(9):1033-49.

17. Rudolph J, Chen L, Majumdar D, Bullock WH, Burns M, Claus T, et al. Indanylacetic Acid Derivatives Carrying 4-Thiazolyl-phenoxy Tail Groups, a New Class of Potent PPAR $\alpha / y / \delta$ Pan Agonists: Synthesis, StructureActivity Relationship and in vivo Efficacy. Journal of Medicinal Chemistry. 2007;50(5):984-1000.

18. Aparoy P, Kumar RK, Reddanna P. Structure and ligand based drug design strategies in the development of novel 5-LOX inhibitors. Current Medicinal Chemistry. 2012;19(22):3763-78.

19. Wassermann AM, Wawer M, Bajorath JR. Activity landscape representations for structure-activity relationship analysis. Journal of Medicinal Chemistry. 2010;53(23):8209-23.

20. Walters WP, Stahl MT, Murcko MA. Virtual screening-an overview. Drug Discovery Today. 1998;3(4):160-78.

21. Brooijmans N, Kuntz ID. Molecular recognition and docking algorithms. Annual Review of Biophysics and Biomolecular Structure. 2003;32(1):335-73.

22. Cheng F, Li W, Liu G, Tang Y. In silico ADMET prediction: Recent advances, current challenges and future trends. Current Topics in Medicinal Chemistry. 2013;13(11):1273-89.

23. Zhang J, Liu X, Xie XB, Cheng XC, Wang RL. Multitargeted bioactive ligands for PPAR $s$ discovered in the last decade. Chemical Biology and Drug Design. 2016;88(5):635-63.

24. Buntrock RE. Chem Office Ultra 7.0. Journal of Chemical Information and Computer Sciences. 2002;42(6):1505-6.

25. Bernstein FC, Koetzle TF, Williams GJ, JrMeyer EF, Brice MD, Rodgers JR, et al. The Protein Data Bank: A computer-based archival file for macromolecular structures. European Journal of Biochemistry. 1977;80(2):319-24.

26. Artis DR, Lin JJ, Zhang C, Wang W, Mehra U, Perreault M, et al. Scaffoldbased discovery of indeglitazar, a PPAR pan-active anti-diabetic agent. Proceedings of the National Academy of Sciences of the United States of America. 2009;106(1):262-7.

27. Kramer B, Rarey M, Lengauer T. Evaluation of the FLEXX incremental construction algorithm for protein-ligand docking. Proteins: Structure, Function and Bioinformatics. 1999;37(2):228-41.

28. Available from: http://www.niper.gov.in/pi_dev_tools/DruLiToWeb/DruLiTo_ index.html.

29. Aiken LS, West SG, Pitts SC. Multiple linear regression. Handbook of Psychology. 2003;481-507.

30. Myers R. Classical and modern regression with applications. Duxbury Press, Boston. 1986

31. Mihalic Z, Nikolic S, Trinajstic N. Comparative study of molecular descriptors derived from the distance matrix. Journal of Chemical Information and Computer Sciences. 1992;32(1):28-37.

32. Maass P, Schulz-Gasch T, Stahl M, Rarey M. Recore: A fast and versatile method for scaffold hopping based on small molecule crystal structure conformations. Journal of Chemical Information and Modeling. 2007;47(2):390-9.

33. Rarey M, Kramer B, Lengauer T, Klebe G. A fast flexible docking method using an incremental construction algorithm. Journal of Molecular Biology. 1996;261(3):470-89.

34. Totrov M, Abagyan R. Flexible protein-ligand docking by global energy optimization in internal coordinates. Proteins: Structure, Function and Bioinformatics. 1997;29(S1):215-20.

35. Lipinski CA. Lead-and drug-like compounds: the rule-of-five revolution. Drug Discovery Today: Technologies. 2004;1(4):337-41.

36. 2-amino-9-[(2S,5R)-3-fluoro-5-methylol-2,5-dihydrothiophen-2-yl]hypoxanthine. 2004. Available from: http://zinc.docking.org/substance/3584559. 
PICTORIAL ABSTRACT

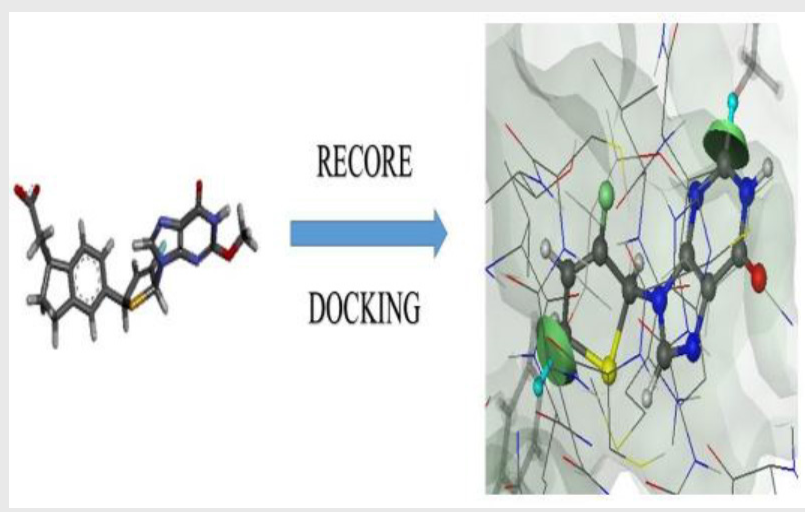

About Authors

\section{SUMMARY}

Diabetes is one of the largest global health emergencies of the $21^{\text {st }}$ century. There are currently various curative treatment available and therapy is reliant on the use of existing anti-diabetic drugs having their own long term toxic effects. Therefore it is crucial for identifying such molecules which are effective and possessing least side effects. Improvement in drug design has become a successively important step and field of interest within healthcare. This article provides an overview and application of potential methods for the enhancement in agonistic activity of PPAR activators and design of novel ligands possessing anti-diabetic characteristics.

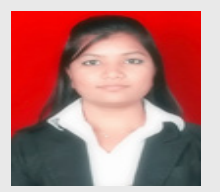

Ms. Neha Verma is Ph.D Research Scholar with research interest in computer aided drug design. She is vigorously involved in researches of molecular docking, molecular dynamics simulation and next generation sequencing. Passionate Investigator of molecular modelling and drug design. The data of the paper are parts of the Ph.D thesis submitted in MANIT, Bhopal supervised by Professor Chouhan.

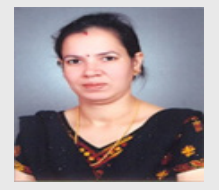

Dr. Usha Chouhan is Associate Professor, Department of Mathematics, Bioinformatics and Computer Applications, MANIT Bhopal, INDIA. She is actively involved in researches of Biomathematics, Computational Biology, Bioinformatics, CADD, Computational Phylogenetics, Soft Computing, etc., published more than 60 research papers in various national and international journals.

Cite this article: Verma N, Chouhan U. Pharmacoinformatic Studies on 4-Thiazolyl-phenoxy Tail Containing Indanyl Acetic Acid Derivatives as PPAR-Pan Agonists as Potent Anti-Diabetic Agent. Indian J of Pharmaceutical Education and Research. 2019;53(3 Suppl 2):s288-s298. 